\title{
Understanding work participation among employees with common mental disorders: What works, for whom, under what circumstances and how? A systematic realist review protocol
}

\author{
Suzanne G.M. van Hees ${ }^{\mathrm{a}, \mathrm{b}, *}$, Bouwine E. Carlier ${ }^{\mathrm{a}}$, Roland W.B. Blonk ${ }^{\mathrm{b}, \mathrm{c}, \mathrm{d}}$ and Shirley Oomens ${ }^{\mathrm{a}, \mathrm{e}}$ \\ ${ }^{a}$ Occupation and health research group, HAN University of Applied Sciences, Nijmegen, The Netherlands \\ ${ }^{\mathrm{b}}$ Tilburg School of Social and Behavioral Sciences, Tilburg University, Tilburg, the Netherlands \\ ${ }^{\mathrm{c}}$ Optentia Research Focus Area, North-West University, Vanderbijlpark, South Africa \\ ${ }^{\mathrm{d}}$ TNO, Leiden, The Netherlands \\ ${ }^{\mathrm{e}}$ Radboudumc, Department of Primary and Community Care, School of Occupational Health, \\ Nijmegen, the Netherlands
}

Received 17 August 2020

Accepted 12 October 2020

\begin{abstract}
.
BACKGROUND: Work participation among employees with common mental disorders (CMDs) is an increasingly important, yet highly complex phenomenon. Given the call for preventing instead of reacting to negative work outcomes, there is a need to understand how employees with CMDs can continue working.

OBJECTIVES: 1) to provide insights in applying a realist approach to the literature review process and 2) to present a way to develop an explanatory framework on work participation, the related causal mechanisms and the interaction with the work context.

METHODS: A systematic realist literature review, using stay at work (SAW) and work performance (WP) as outcomes of work participation. This protocol paper explains the rationale, tools and procedures developed and used for identification, selection, appraisal and synthesis of included studies.

RESULTS: The review process entailed six steps to develop so called 'middle range program theories'. Each step followed a systematic, iterative procedure using context-mechanism-outcome (CMO) configurations.

CONCLUSIONS: Conducting a realist review adds on the understanding to promote work participation, by examining the heterogeneity and complexity of intervention- and observational studies. This paper facilitates other researchers within the field of occupational health by demonstrating ways to develop a framework on work participation using realist synthesis.
\end{abstract}

Keywords: Realist research, methodology, occupational health, mental disorders

*Address for correspondence: Suzanne van Hees, Kapittelweg 33, P.O. Box 6960, 6503 GL Nijmegen, The Netherlands. Tel.:
+31611742858; E-mail: Suzanne.vanhees@han.nl.; ORCID ID: 0000-0002-0281-7909 


\section{Introduction}

Work participation among employees with common mental disorders (CMDs) is an increasingly important topic because it has positive consequences for the employee's social status and health $[1,2]$ and for organizations and society as a whole [3]. Being at work contributes to wellbeing and mental health [4, 5]. Alternatively, being depressed, anxious or stressed affects the working life of the employee in terms of negative work outcomes, such as sickness absence and impaired work performance [6, 7]. It is estimated that at any point in time, one-sixth of the working age population is suffering from CMDs [3, 8, 9]. CMDs cause the highest number of sickness absence and reduced work capacity in the Western world $[10,11]$. Considering the magnitude of CMDs, the Organisation for Economic Co-operation and Development (OECD) calls for preventing instead of reacting to negative work outcomes from poor mental health. However, there is rather limited evidence on how to promote work participation despite CMDs, the related causal mechanisms and the complex interaction with the work context $[3,8,12]$. To promote work participation, it is important to understand what occurs among those employees with CMDs who continue working.

Work participation is defined as the way an employee fulfils the work role in the workplace [1, 7]. This study gives attention to employees who actually continue working instead of being sick listed, either "successfully" or "struggling", and how it affects their work performance. In this study, work participation is operationalized by those two work outcomes, that are linked to our understanding of work participation among employees with CMDs [7, 13]. The first outcome is stay at work (SAW), that is, 'the employee is currently working'. SAW is a relatively new concept in the field of occupational health and is not uniformly defined in the literature [14]. A diversity of terms has been used so far, such as staying at work $[14,15]$, refraining from sick leave despite the experience of mental illness [16] or absence of absenteeism [14, 17]. We define SAW as continued working, indicated as no absenteeism or not being absent for more than $50 \%$ or no longer than 6 weeks $[7,17,18]$. Besides SAW, we are interested in the way CMDs affect employees' work performance (WP), or 'how the employee functions at work'. This second outcome is indicated by the level of diversion in WP or indicated by presenteeism [19]. CMDs refer to depression and anxiety disorder as the most frequent disorders [9], but other mental disorders such as adjustment disorders and burnout are also included [2, 20]. A large number of employees who suffer from CMDs are undiagnosed and do not receive treatment [21], or do not disclose their symptoms of mental illness at work [22]. Those employees may struggle while they continue working due to an imbalance between abilities and demands, referred to as "work instability" [23]. However, they have not (yet) consulted a psychiatrist, occupational physician or general practitioner. Therefore, diagnosis alone may not be sufficient to understand work participation with a CMD in terms of functional limitations appearing at the workplace $[24,25]$. Consequently, our study population consists of either employees with clinically diagnosed CMDs [26] or employees with self-reported psychological complaints such as reduced concentration, irritation, fatigue or gloom [24]. Since most people affected by CMDs or psychological complaints are employed and actually working, this phase whilst being at work needs increased attention.

In our attempt to answer the question What works to continue working?, we suppose that factors promoting work participation among employees with CMDs are known to some extent. Reviews on work participation among employees with CMDs reveal that staying at work and being productive is affected by individual factors such as higher symptom severity (e.g. a past history of absenteeism, co-morbidity), and work-related factors (e.g. high job demands, lower job control) [1, 7]. In practice, efforts to promote work participation are often taken, such as psychotherapeutic treatment, to work part-time temporarily or to decrease the work load [18]. However, it remains unclear What really makes work work? For example, how do employees try to manage their tasks while feeling depressed or anxious? What efforts are needed to promote the fit between the employee's abilities, tasks and the particular work context is not fully understood [1]. Promoting work participation is complicated and challenging; and therefore requires a thorough understanding in order to act upon it. However, an explanatory framework on work participation with CMDs so far is lacking.

Whilst there seems little available knowledge on work participation of employees with CMDs that remain at work, there is substantial knowledge in the field of occupational health among employees with CMDs who were sick listed and in return-towork trajectories $[2,17,18,20,27,28]$. In those review studies, the dynamics between individual 
factors, work-related factors and the work environment have been proven crucial and highly complex. Some empirical research has shown that the hidden, work-related factors such as sense of belonging, openness of the manager and a supportive relationship between managers and employees are factors that promote return to work $[12,29]$. As return-towork can be considered as a complex multifactorial process, likewise the phase of working with CMD can be characterized as a dynamic interactive phenomenon [18]. Although we can learn from those studies in other phases, such as returning to work, we have not yet understood sufficiently how employees with CMDs can continue working. Hence, there is a need for a theoretical framework on the complexity of factors influencing work outcomes of work participation, and their underlying mechanisms, in order to develop effective workplace interventions [13, 30-32].

Beyond understanding which mechanisms lead to work participation, we attempt to understand under what (work) circumstances those mechanisms occur. Reviews on workplace mental health interventions revealed that studies often do not address organizational- or work factors, thereby missing the complexity of the social context in the work environment [21, 31-33]. Nevertheless, some studies have shown that such interventions and its effectiveness highly depend on the context in which they are implemented [34-36]. For instance, Cullen et al. (2018) indicate that the suggestion given by a personal coach for an employee to apply for a work modification is more likely to be taken up in an appropriate, supportive organizational culture [35]. Since interventions are often implemented and evaluated in highly complex organizational contexts, capturing under what circumstances those interventions work deserves rigorous investigation $[12,30]$.

In response to the lack of a theoretical framework on work participation among employees with CMDs, we propose the Capability-for-Work model. We are interested to know if we can apply a heuristic model from a related concept in occupational health, namely to maintain employment among older employees. The Capability-for-Work model defines capabilities as functioning that the person is able to achieve, depending on his or her particular circumstances [37]. This model incorporates various personaland environmental (work) conditions, which enable employees to convert personal- and work inputs into work capabilities [38]. Also, it reflects the complex interaction of multiple personal- and work factors and its emphasis on the vast complexity of the work context [38].

Given the lack of evidence base on mechanisms and the (work) context to understand the complexity of work participation, we argue that it is needed to move from What works, to What works, for whom, under what circumstances and how [39]. We aim to fill this knowledge gap by conducting a systematic literature review, contributing to the building of explanatory program theories. Earlier reviews assessed literature in a traditional way, by separately reporting personal- and work-related factors or the effect of its interventions [7, 8, 21, 32, 33, 40]. However, the assessment of outcomes of interventions remains weak, partly because the methodologies that were used did not grasp the complexity of the (work) circumstances or did not address mechanisms of change $[8,32,33,40]$. Additional factors to the intervention itself, including circumstances related to the organizational structure, and mechanisms related to interpretations and efforts, will affect the effectiveness of the intervention [12]. Evaluation studies such as randomized controlled trials (RCTs) are designed to show us whether the intervention works (or not), and do not provide information on which of the intervention activities (planned or unplanned) led to improvements or in what context those improvements occurred [39]. Furthermore, interventions in everyday practice are not controlled by trial protocols. There are calls for more rigorous evaluations that go beyond the identification of efficacy in a controlled evaluation setting, so it can inform policy and practice in terms of factors that may promote work participation in diverse workplaces [13]. Realist research may provide a methodological answer to develop a theoretical framework [41]. It is a theory-driven evaluation method that is designed for complex social interventions or phenomena, providing an analysis that is more explanatory in nature $[42,43]$. Both the need for theory building on the evidence base of work participation for employees with CMDs and the diversity of its measured outcomes underpin our rationale for conducting a systematic realist literature review (SRLR).

To our knowledge, a realist review on work participation among employees with CMDs has not been done before. The current paper reports on the protocol of a SRLR. The objective of this study is two-fold: 1) to provide insights in applying a realist approach to the review process, including the rationale, tools and procedures developed and used for identification, selection, appraisal and synthesis of included studies, 
and 2) to present a way to develop a framework with explanatory program theories on the emerging theme of work participation, among employees with CMDs.

\section{Materials and methods}

\subsection{Rationale for systematic realist literature review}

Realist review was developed by Pawson et al. (2005) from the philosophical traditions of critical realism, which seeks to consider the complexity of causal relations when explaining social interactions and interventions [42]. The aim of a realist synthesis of the literature is not simply to answer the question: "Does this intervention work?" but to answer a more complex series of questions: "What is it about this intervention that works? In what circumstances does this intervention work or not? For whom does the intervention work?" [41]. A realist review does this by unpacking the theories embedded in descriptions of interventions, and by looking for the impact of context on the effectiveness of interventions [42]. Whereas, post-positivism researchers use the empirical testing approach to understand what works, the realist approach involves an ongoing interpretative process to configure context, mechanisms and outcomes [41]. More technically, realist synthesis searches for common underlying mechanisms that occur under what circumstances, also called context-mechanism-outcome (CMO) configurations. Causality can only be suggested if there is an understanding of the context $(\mathrm{C})$ in which a particular mechanism (M) generates an outcome (O) [41, 43]. These CMO configurations regarding the outcomes SAW and WP can be retrieved from the realist synthesis, leading to so-called middle range program theories [44]. This approach allows us to examine the diversity and complexity of observational and intervention studies reporting on the outcomes SAW and WP simultaneously, as well as quantitative and qualitative studies.

\subsection{Definition of realist terms}

Middle range program theories: theories that lie between the working hypotheses from the researchers who design and evaluate an intervention and the all-inclusive systematic efforts to develop a coherent theory that may explain all of the observed uniformities of social behaviour, social organization and social change [45].

Context: context refers to "something that enables or disables the current mechanism of interest to fire" (p.54) [45]. It often refers to the 'setting' of programs and research. As conditions change over time, the context may also reflect aspects of those changes while the program is implemented.

Mechanisms: mechanisms are underlying entities, processes or structures that lead to influence the outcome [46]. This can refer to processes within the participant of an intervention or exposure, his or her cognitive and emotional responses, typically related to the intervention or exposure being offered. But it can also refer to the context, like a company in which the participant is working.

Outcome: an outcome is what can be measured in terms of impact across the target population, using measurable or measured indicators. Outcomes can be considered as quantitative or qualitative, and intended or unintended [44]. Realist research combines the strength of both research paradigms, in which qualitative studies may provide causal explanations of mechanisms, whereas quantitative studies may distinguish regularities, patterns, and features of the population groups.

$C M O$ configuration: describes the causal links between context, mechanisms and outcome considered as causative explanations pertaining to the evidence on the topic of interest [45].

\subsection{Procedures}

The SRLR follows the steps and procedures outlined by RAMESES publication Standards for Realist Synthesis in an iterative manner [47] (refer to Fig. 1). Regarding the search strategy (step 2 ), in which we conducted a systematic search of the literature, we adhere to the PRISMA guidelines for the conduct of systematic reviews [48]. Details of the protocol for this SRLR are registered on PROSPERO and can be accessed at https:// www.crd.york.ac.uk/prospero/display_record.php?R ecordID=108913. Our team consisted of researchers from various disciplines, namely public health, occupational health, sociology and psychology. As recommended in realist research, having a multidisciplinary team helped to shape our definitions and approach described in the protocol [41]. 


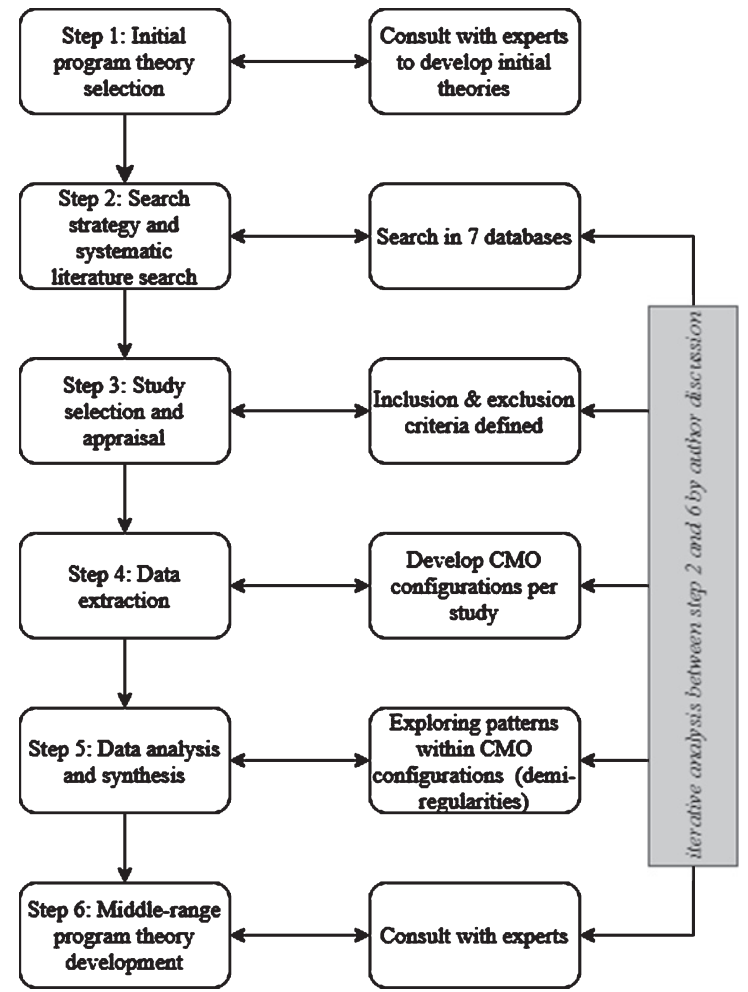

Fig. 1. Overall realist review process.

\section{Results of the followed steps}

\subsection{Step 1: select initial program theory}

The overall focus in this SRLR is to select, test and ultimately revise an initial program theory, using academic literature. By drawing middle range program theories, we develop an explanatory framework on both outcomes of work participation, SAW and WP. Given the complexity of work participation and the unknown scope and nature of the evidence base beforehand, we found it useful to initially define an initial program theory using a conceptual model. Van Der Klink et al. (2016) identified a conceptual model based on the concept of capability [37]: the Capability-for-Work model [38]. It incorporates the following work capabilities: 1) the use of knowledge and skills, 2) the development of knowledge and skills, 3) involvement in important decisions, 4) building and maintaining meaningful contacts at work, 5) setting own goals, 6) having a good income and 7) contributing to something valuable [38]. We assume that work participation is determined by the way an employee succeeds in converting a combination of inputs and resources into capabilities and subsequently into valuable work functioning. Inputs are the personal resources (e.g. health, knowledge) or workplace resources (e.g. a set of tasks) and conversion factors refer to the process of converting one's inputs to tangible capabilities, resulting into work functioning that the employee chooses to achieve. Work functioning is defined and assessed in our study as the employee is currently working (SAW) and how the employee functions at work (WP) (refer to Fig. 2). We explore how the Capability-for-Work model could help us to interpret and summarize our findings. It challenges us to identify and order personal and contextual inputs and conversions from the set of retrieved mechanisms and contextual factors extracted from the selected studies. Those pre-existing conditions/circumstances, referred to as context, may act on macro level (existing public policy, historical, geographical, socio-political and labour market conditions), meso level (organization, staffing and leadership, cultural norms and values, physical and social environment at the workplace) and micro level (personal environment/personal resources and social environment of the employee). As suggested by Corbiere et al. [13], we also explore how the work outcome of Work performance (WP) possibly acts as a capability to achieve the work outcome of (the choice to) stay at work (SAW).

\subsection{Step 2: developing a search strategy and literature search}

We performed an electronic search in June 2020 in the following databases, Pubmed, Medline, PsycInfo, Embase, Cochrane, Cinahl, Web of Science, searching for scientific peer-reviewed studies describing factors or mechanisms on both SAW and WP for employees with CMDs. A total of 4,238 citations were retrieved. As from the end of the 20th century, mental health at the workplace gained increasing interest. Therefore, studies from the year 1995 and onwards were included. This search strategy captured SAW and WP in academic journals of various disciplines, including occupational health science, human resource management, organizational psychology, social sciences and medical sciences. In consultation with a health research librarian, we developed a search string and trialled iteratively (Supplement). We used a combination of three groups of keywords, that is, employees with common mental disorders, stay at work or (reduced) work performance to search databases. These groups of keywords 


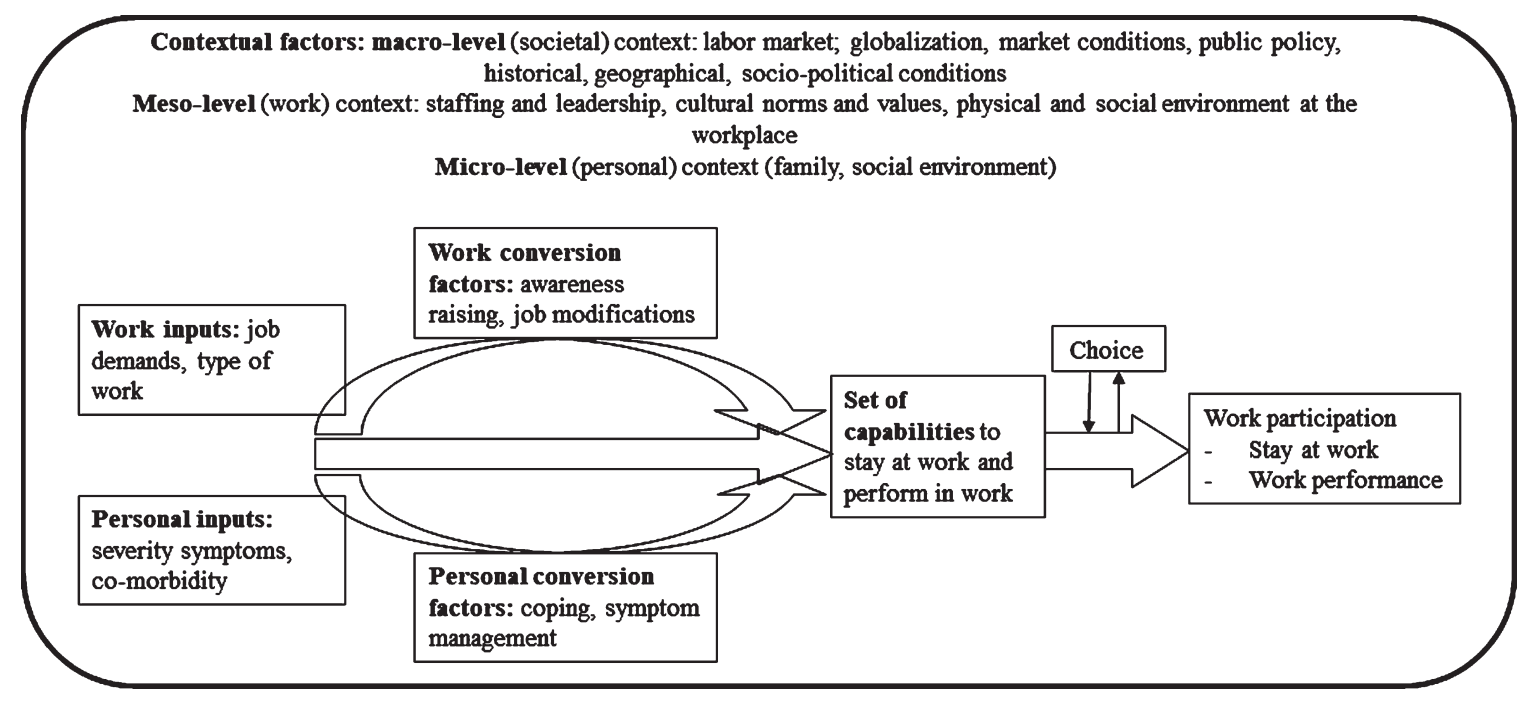

Fig. 2. Model of the initial program theory on work participation, based on the Capability-for-Work model [38].

consisted of search terms from all seven databases: mesh terms (PubMed), thesaurus (psychInFO), and heading terms (CINAHL). Also, synonyms and free text words were used.

\subsection{Step 3: study selection and appraisal}

\subsubsection{Selection and inclusion criteria}

Titles and abstracts were imported into EndNote and duplicate references were removed. Thereafter, all references were imported into the software of Rayyan [49]. Relevant screening consisted of two steps. First, two researchers dually assessed the studies' relevance with inclusion and exclusion selection criteria during the title and abstract screening (refer to Table 1). This step led to a selection of 191 citations, from the 2,235 citations after removal of duplications. Next, two independent researchers ( $\mathrm{SH}$ and BC; SH and EV) dually read the full texts and decided whether articles should be included for data extraction. We based the decisions on the selection criteria as well as whether the findings contribute to theory testing of the initial program theory and its refinement and thus contain contexts, mechanisms and outcomes of interest.

\subsubsection{Quality appraisal}

We used the Mixed Methods Appraisal Tool to appraise the methodological quality (the rigor) of quantitative studies, mixed method studies and qualitative studies [50]. This tool contains of two screening questions and 25 items for appraising the methodological quality of five categories of studies: qualitative studies, RCTs, non-randomized studies, quantitative descriptive studies and mixed methods studies [51]. Two independent research teams conducted the quality appraisal. We used studies of high quality (rated green) to form CMO configurations. Studies with medium quality (rated orange) containing possible informative mechanisms were only used to support CMO configurations derived from high quality studies. Studies which did not define a clear research question or in which collected data did not allow us to address the research question (answer a 'no' to screening questions, rated red) were excluded. After full text screening and quality appraisal, 61 articles were included.

\subsection{Step 4: data extraction}

In realist review, data extraction includes descriptions and explanations of how and why the mechanisms may (or may not) be triggered in a particular context, with regard to the selected outcomes [47]. We drew up a digital data extraction form in order to record study information, including study characteristics (e.g. methodology, sample size), contexts, mechanisms and outcomes. For each study, we drafted one or more CMO configurations (refer to Table 2). These configurations described how contextual factors (characteristics of employees with CMDs and their context) and mechanisms (human processes or responses) led to the desired outcome (SAW or WP). If mechanisms or contextual factors 
Table 1

Inclusion and exclusion criteria

\begin{tabular}{lc}
\hline Inclusion criteria & Exclusion criteria \\
\hline Primary outcome: stay at work, absence of & $\begin{array}{c}\text { Studies including a general } \\
\text { population of employees, and their } \\
\text { absenteeism, continue working: subjects had to }\end{array}$ \\
perform paid work, either part time or fulltime. If & primary stress prevention (not providing \\
recorded as sick, subjects had to work for at least 50\% & subgroups with employees at risk) \\
within the first 6 weeks after their first sick day & Where subpopulations of \\
Secondary outcome: quality of work: such as, & employees with CMDs were not \\
presenteeism, reduced or impaired work capacity, & taken as subpopulation in the data analysis \\
work performance or workability & All severe mental disorders and \\
Employees having one or more common mental disorders, or employees having & personality disorders \\
symptoms of mental health problems, who 'struggle at work', assessed & Study on sickness absence, and \\
with self-assessment tools. & thus reporting on employees on \\
If burnout score is based on the Maslach burnout & sick leave rather than still at work. \\
inventory: only when sub analyses are done on the & Economic impact studies \\
score on emotional exhaustion & \\
Individuals aged between 18 and 65 years & \\
Geographical/economic scope: at first: globally. & \\
Study design a primary research study and published & \\
in peer-reviewed journals, reporting randomized & \\
controlled trials, cohort, case-control or cross- & \\
sectional studies, or qualitative descriptive & \\
(case) studies. & \\
Published in English, from 1995 and onwards &
\end{tabular}

were retrieved on the dichotomous outcome of SAW (yes or no), or reported the reduced chance or risk on absenteeism or sick leave, then we converted this factor into a facilitator to stay at work. Thereafter, we imported the CMO configurations of each study in Excel, in order to develop a structured data collection format, ordering studies by outcome (SAW and/ or WP) and type of study (observational or interventional). Two independent research teams discussed the results, for cross checking to identify any inconsistencies or inaccuracies. From the 61 articles, 46 (75\%) articles described outcomes regarding SAW and $41(67 \%)$ articles reported about WP.

\subsection{Step 5: data analysis and synthesis}

We performed analyses by exploring patterns within these CMO configurations by thematic analysis, that led to middle range program theories [44]. Firstly, after preliminary annotating and extracting data, studies showing similar CMO configurations were coded as barriers or facilitators, regarding each outcome. Those CMO configurations were sorted according to common themes in occupational health and refined in terms of mechanisms. Then, those configurations, categorized in themes, were embedded in a larger chronology of the outcomes, to identify and explain causal effects. Patterns of outcomes (also called demi-regularities) were identified and sorted, using 'if ..., then ...' statements). Using the thematic analysis leading to demi-regularities, we synthesized mainly from qualitative studies the various mechanisms that occur and under what circumstances those would lead to the outcome. The quantitative studies provided mainly CMO configuration containing of one mechanism, explaining its causal relationship with the outcome. Figure 3 shows an example of a CMO configuration, in which context, mechanism and outcome were distinguished, based on the Capability-for-Work model (initial program theory).

\subsection{Step 6: revised program theory}

The final step of the synthesis will provide an explanatory framework for how, why and under what circumstances employees with CMDs stay at work and maintain their level of work performance. Hence, this step consists of confirming or modifying demiregularities with the initial program theory, leading to middle range program theories. We are currently conducting this last step. We will choose demiregularities that were based on patterns observed in the CMO configurations of step 5. This process of confirming or modifying includes testing the Capability-for-Work model as an initial program 
Table 2

Example of context-mechanisms-outcome configuration

\begin{tabular}{lcc}
\hline Context & Mechanism & $\begin{array}{l}\text { Outcome relevant for study } \\
\text { (Stay at work or work performance) }\end{array}$ \\
\hline 11 Finnish male workers & Personal feedback and group & Positive effect on workability \\
with stress and mood & meetings and participation in & \\
problems, versus 12 on & intervention decreased depressive & \\
waiting list [53]. & symptoms so workability increases. & \\
\hline
\end{tabular}

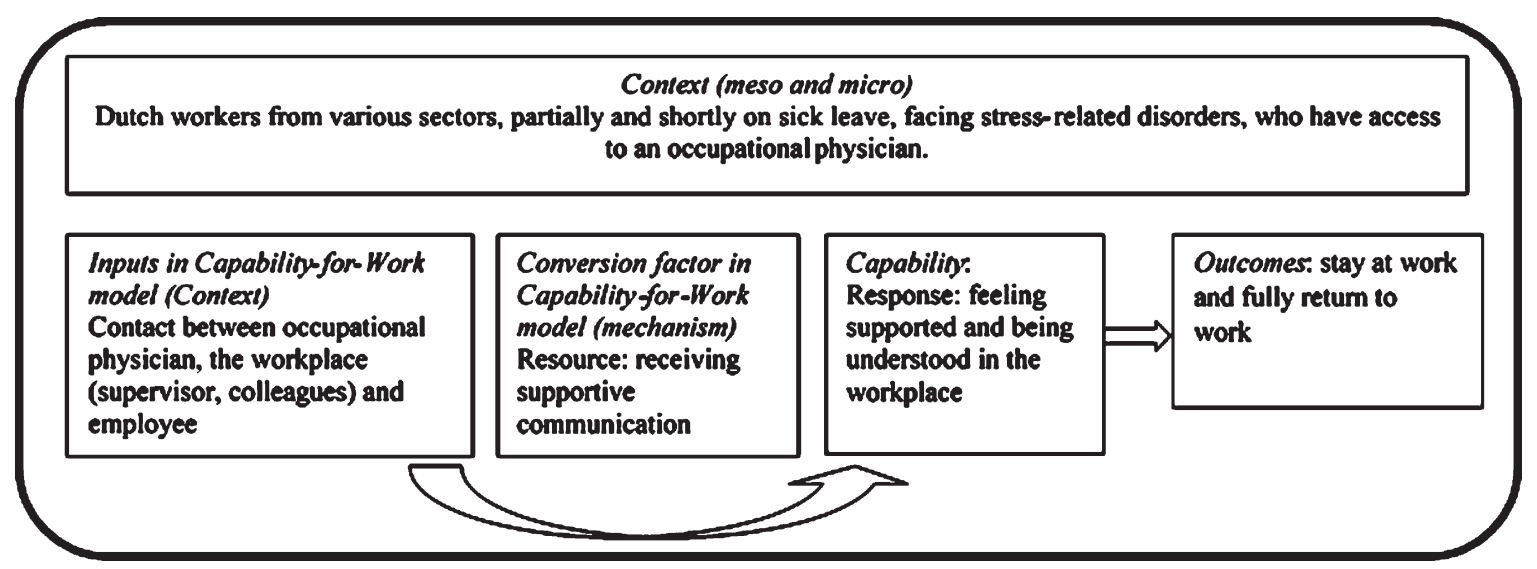

Fig. 3. Example of CMO configuration, using the initial program theory [18].

theory as well as possible other candidate theories to explain or understand patterns. Accordingly, the analytic process involves iterative testing and refinement of theoretically-based explanations [47]. Discussion in our research team ensures soundness and consistency in the analytic process of demi-regularities and development of the middle range program theories.

\section{Discussion}

Work participation of employees with common mental disorders is one of the key challenges in occupational health. Efforts to support those employees to continue working are important for individuals, organizations and the society as a whole, though often complex and challenging. Understanding work participation requires a rigorous approach. This review protocol provides useful insights on how to apply a realist synthesis in theory building on a complex phenomenon such as work participation among employees with CMDs. Besides, this paper demonstrates the application of a heuristic model to further understand work participation and therefore a new way to build on existing theory. By using realist synthesis methods, we develop an explanatory framework resulting in a contextual understanding of mechanisms of work participation. Findings from the ongoing review are forthcoming. Subsequently, we will test this framework with various stakeholders, such as employers (line managers), employees with CMDs and several occupational health professionals. By testing and elaborating the program theories, we expect to know which conditions are necessary for the mechanisms to be triggered [39]. This will result in strategies that are effective, efficient, and have a potential for successful implementation in daily practice. Those insights could help employers, policy-makers and researchers in the development of evidence-based interventions.

To the best of our knowledge, this is one of the first studies using realist research in the field of occupational health [39]. This study thereby adds to academic literature on the use of a realistbased approach in the examination of evidence-based organizational occupational health interventions. Traditional systematic reviews might miss out on hidden mechanisms or on its factors interacting within a particular setting or context [7, 12, 39]. Realist synthesis allows us to examine the heterogeneity and complexity of various studies reporting on the selected work outcomes, which led to a richer set of data. Also, based on the review process thus far, we suppose that realist synthesis may offer a deeper 
understanding of a wide range of factors and their interaction with the workplace. This protocol paper provides a step-wise approach on how to draw out patterns from such a diverse dataset, and advancing knowledge regarding conducting a realist review and develop program theories. This facilitates researchers to gain insights into the application of realist research in the field of occupational health and enhance the interpretation and critical examination of our review findings [47].

This is one of the first studies that investigates mechanisms and contexts of employees with CMDs who continue working. This phase of stay at work in which the employee continues working focuses on the prevention of negative work outcomes. Given the relatively new concept of stay at work we choose for a systematic literature search, to achieve an overview, a level of uniformity and completeness regarding the evidence base covering employees with CMDs who are currently working. This paper adds on the conceptualization of stay at work by reporting our initial program theory, definitions of outcomes and, inclusion and exclusion criteria. Accordingly, we attempt to stimulate the debate among researchers on the understanding of work participation.

Underpinned and informed by an innovative model used in occupational health, this study provides insights on how to develop an explanatory framework on work participation for employees with CMDs. We used the Capability-for-Work model as an initial program theory to discover patterns of outcomes leading to middle range program theories. This model seems useful in exploring patterns, considering a plethora of CMO configurations retrieved from data extraction. Consequently, it helps us to grasp the complexity of work participation. Because work participation is such a multifactorial phenomenon due to the important role of the work context, it deserves a model that can handle such complexity. Furthermore, understanding work participation through a different lens, namely of work capabilities, may add on theory development of this model for other, related work outcomes.

We have anticipated the following challenges common to many realist reviews. Firstly, because we work with published research, with predefined variables and measurements, we gain insights in how contextual factors, mechanisms, and outcomes have been measured and reported to date. However, often mechanisms are hidden and not explicitly described in the results section, resulting in a lack of in-depth information. Hence, we used information regarding context, mechanisms or implementation from the discussion section in publications, to capture more closely what is important in those mechanisms according to the authors [45]. Secondly, retrieved factors were defined as mechanisms by some studies while other studies identified those factors as context or circumstances. This challenge refers to the so called 'ripple effect', in which a certain factor was configured as an aspect of the context, being a precondition, and other times as a mechanism [52]. We responded to this challenge by incorporating the ripple effect within the program theory and by adhering to our presented definitions of context and mechanism [46]. Thirdly, it was challenging to select and appraise studies on relevance and rigor. Since we allowed heterogeneity in the type of studies and measures of outcomes, this led to a variety $\mathrm{CMO}$ configurations with different levels of relevance or rigor. To overcome this, we used a systematic approach by two independent researchers in each step, using clearly defined concepts and inclusion and exclusion criteria to ensure relevance. In addition, we used the MMTA, a standardized tool for quality appraisal, to ensure rigor.

Possible limitations in our study are related to the design of the SRLR. Realist researchers acknowledge that there is not one way of conducting a realist review [41]. As other review approaches may demand a systematic approach, realist review process is ultimately iterative and flexible, however transparent. Nevertheless, we decided to conduct a systematic database search, based on the limited evidence base of our topic of interest. Despite our sound reasons for the systematic approach, we did not use an iterative process of selecting other types of references than scientific peer-reviewed studies, thus in step 2 our review may not be a typical realist review. Furthermore, in step 5 , we searched and examined ideas about the causal factors linked to the evidence, using CMO-statements which lead to demi-regularities [44]. However, we came across patterns or mechanisms without any causative explanations pertaining to the evidence. Those gaps will guide us to identify areas for further research. To overcome those limitations we report our selection, appraisal and analysis protocol adding to transparency and replicability of our review process.

\section{Conclusion}

In conclusion, given the complexity to promote work participation, conducting a realist review adds on the understanding of the dynamics between 
personal- and work factors, the underlying mechanisms of work participation and the (work) context. Realist synthesis provides a way to gain insights into how work outcomes, such as staying at work or work performance in reality evolve. Our initial program theory, the Capability-for-Work model helps to explain how and when retrieved mechanisms and interventions lead to those work outcomes. This paper facilitates other researchers within the field of occupational health by demonstrating ways to develop a framework on work participation using realist synthesis. Findings from this SRLR will result in an explanatory framework on work participation among employees with CMDs. The framework will enable us to better understand how workplace interventions achieve the desired outcomes through evidence-based practices.

\section{Acknowledgments}

Thanks are due to Thomas Pelgrim for his support to develop a librarian-guided search string, to Jac van der Klink, founder and expert of the Capability-forWork model for his contribution the discussion and application of this model in our study and to Emma Vossen (EV) as co-researcher, by supporting on study selection and appraisal.

\section{Author contributions}

All authors contributed to the study conception and design. Suzanne van Hees and Bouwine Carlier performed the literature search and data analysis. The first draft of the manuscript was written by Suzanne van Hees and all authors commented on previous versions of the manuscript. All authors read and approved the final manuscript.

\section{Conflict of interest}

The authors declare that they have no conflict of interest.

\section{Funding}

This work was supported by GAK Institute (grant number 2017-928).

\section{Supplementary material}

The supplement is available from https://dx. doi.org/10.3233/WOR-213515.

\section{References}

[1] Thisted CN, Nielsen CV, Bjerrum M. Work Participation Among Employees with Common Mental Disorders: A Meta-synthesis. J Occup Rehabil. 2018;28(3):452-64.

[2] de Vries H, Fishta A, Weikert B, Rodriguez Sanchez A, Wegewitz U. Determinants of Sickness Absence and Return to Work Among Employees with Common Mental Disorders: A Scoping Review. J Occup Rehabil. 2018;28(3):393-417.

[3] OECD. Sick on the Job?: Myths and Realities about Mental Health and Work. Paris: OECD Publishing; 2012.

[4] Lotters F, Carlier B, Bakker B, Borgers N, Schuring M, Burdorf A. The influence of perceived health on labour participation among long term unemployed. J Occup Rehabil. 2013;23(2):300-8.

[5] Waddell G, Burton AK. Is work good for your health and wellbeing? London: The Stationery Office; 2006.

[6] Steel Z, Marnane C, Iranpour C, Chey T, Jackson JW, Patel $\mathrm{V}$, Silove D. The global prevalence of common mental disorders: a systematic review and meta-analysis 1980-2013. Int J Epidemiol. 2014;43(2):476-93.

[7] Lagerveld SE, Bultmann U, Franche RL, van Dijk FJ, Vlasveld MC, van der Feltz-Cornelis CM, Bruinvels DJ, Huijs JJ, Blonk RW, van der Klink JJ, Nieuwenhuijsen K. Factors associated with work participation and work functioning in depressed workers: a systematic review. J Occup Rehabil. 2010;20(3):275-92.

[8] Harvey S, Joyce S, Tan L, Johnson A, Nguyen H, Modini M, Groth M. Developing a mentally healthy workplace: a review of the literature. Australia: National Mental Health Commission and the Mentally Health Workplace Alliance; 2014.

[9] Ormel J, Petukhova M, Chatterji S, Aguilar-Gaxiola S, Alonso J, Angermeyer MC, Bromet EJ, Burger H, Demyttenaere K, de Girolamo G, Haro JM, Hwang I, Karam E, Kawakami N, Lepine JP, Medina-Mora ME, Posada-Villa J, Sampson N, Scott K, Ustun TB, Von Korff M, Williams DR, Zhang M, Kessler RC. Disability and treatment of specific mental and physical disorders across the world. Br J Psychiatry. 2008;192(5):368-75.

[10] Murray CJL, Vos T, Lozano R, Naghavi M. Disabilityadjusted life years (DALYs) for 291 diseases and injuries in 21 regions, 1990-2010: a systematic analyis for the Global Burden of Disease Study 2010. Lancet. 2012;380(21972223).

[11] Harvey S, Henderson M, Lelliott P, Hotopf M. Mental health and employment: much work still to be done. Br J Psychiatry. 2009;194(3):201-3.

[12] Higgins A, O'Halloran P, Porter S. Management of long term sickness absence: a systematic realist review. J Occup Rehabil. 2012;22(3):322-32.

[13] Corbiere M, Negrini A, Dewa CS. Mental Health Problems and Mental Disorders: Linked Determinants to Work Participation and Work Functioning. 2013. In: Handbook of Work Disability: Prevention and Management [Internet]. New York: Springer Science Business Media 
[14] de Vries H, Reneman MF, Groothoff JW, Geertzen JH, Brouwer S. Factors promoting staying at work in people with chronic nonspecific musculoskeletal pain: a systematic review. Disabil Rehabil. 2012;34(6):443-58.

[15] Howard KJ, Mayer TG, Gatchel RJ. Effects of presenteeism in chronic occupational musculoskeletal disorders: stay at work is validated. J Occup Environ Med. 2009;51(6): 724-31.

[16] Duijts SF, Kant I, van den Brandt PA, Swaen GM. Effectiveness of a preventive coaching intervention for employees at risk for sickness absence due to psychosocial health complaints: results of a randomized controlled trial. J Occup Environ Med. 2008;50(7):765-76.

[17] Dekkers-Sanchez PM, Hoving JL, Sluiter JK, Frings-Dresen $\mathrm{MH}$. Factors associated with long-term sick leave in sicklisted employees: a systematic review. Occup Environ Med. 2008;65(3):153-7.

[18] Noordik E, Nieuwenhuijsen K, Varekamp I, van der Klink JJ, van Dijk FJ. Exploring the return-to-work process for workers partially returned to work and partially on long-term sick leave due to common mental disorders: a qualitative study. Disabil Rehabil. 2011;33(17-18):1625-35.

[19] Burton WN, Chen CY, Conti DJ, Schultz AB, Pransky G, Edington DW. The association of health risks with onthe-job productivity. J Occup Environ Med. 2005;47(8): 769-77.

[20] Andersen MF, Nielsen KM, Brinkmann S. Meta-synthesis of qualitative research on return to work among employees with common mental disorders. Scandinavian Journal of Work, Environment \& Health. 2012;38(2):93-104.

[21] Pomaki G, Franche RL, Murray E, Khushrushahi N, Lampinen TM. Workplace-based work disability prevention interventions for workers with common mental health conditions: a review of the literature. J Occup Rehabil. 2012;22(2):182-95.

[22] Brouwers EPM, Joosen MCW, van Zelst C, Van Weeghel J. To Disclose or Not to Disclose: A Multi-stakeholder Focus Group Study on Mental Health Issues in the Work Environment. J Occup Rehabil. 2020;30(1):84-92.

[23] Danielsson L, Elf M, Hensing G. Strategies to keep working among workers with common mental disorders - a grounded theory study. Disabil Rehabil. 2019;41(7):786-95.

[24] Main CJ, Shaw WS, Hopkinton Conference Working Group on Workplace Disability P. Employer Policies and Practices to Manage and Prevent Disability: Conclusion to the Special Issue. J Occup Rehabil. 2016;26(4):490-8.

[25] OECD. Organization for Economic Co-operation and Development. Fit Mind, Fit Job. From Evidence to Practice in Mental Health and Work. Paris: OECD Publishing; 2015.

[26] American Psychiatric Association. Diagnostic and statistical manual of mental disorders. 5th ed. Arlington: American Psychiatric Publishing; 2013.

[27] Salomonsson S, Hedman-Lagerlof E, Ost LG. Sickness absence: a systematic review and meta-analysis of psychological treatments for individuals on sick leave due to common mental disorders. Psychol Med. 2018;48(12):1954-65.

[28] Villotti P, Gragnano A, Lariviere C, Negrini A, Dionne CE, Corbiere M. Tools Appraisal of Organizational Factors Associated with Return-to-Work in Workers on Sick Leave Due to Musculoskeletal and Common Mental Disorders: A Systematic Search and Review. J Occup Rehabil. 2020.

[29] MacEachen E, Clarke J, Franche RL, Irvin E, Workplacebased Return to Work Literature Review G. Systematic review of the qualitative literature on return to work after injury. Scand J Work Environ Health. 2006;32(4):257-69.

[30] Rugulies R, Aust B. Work and mental health: what do we know and how can we intervene? Scand J Work Environ Health. 2019;45(6):529-32.

[31] McDowell C, Fossey E. Workplace accommodations for people with mental illness: a scoping review. J Occup Rehabil. 2015;25(1):197-206.

[32] Furlan AD, Gnam WH, Carnide N, Irvin E, Amick BC, 3rd, DeRango K, McMaster R, Cullen K, Slack T, Brouwer S, Bultmann U. Systematic review of intervention practices for depression in the workplace. J Occup Rehabil. 2012;22(3):312-21.

[33] Joyce S, Modini M, Christensen H, Mykletun A, Bryant R, Mitchell PB, Harvey SB. Workplace interventions for common mental disorders: a systematic meta-review. Psychol Med. 2016;46(4):683-97.

[34] Corbiere M, Shen J, Rouleau M, Dewa CS. A systematic review of preventive interventions regarding mental health issues in organizations. Work. 2009;33(1):81-116.

[35] Cullen KL, Irvin E, Collie A, Clay F, Gensby U, Jennings PA, Hogg-Johnson S, Kristman V, Laberge M, McKenzie D, Newnam S, Palagyi A, Ruseckaite R, Sheppard DM, Shourie S, Steenstra I, Van Eerd D, Amick BC, 3rd. Effectiveness of Workplace Interventions in Return-toWork for Musculoskeletal, Pain-Related and Mental Health Conditions: An Update of the Evidence and Messages for Practitioners. J Occup Rehabil. 2018;28(1):1-15.

[36] Fridrich A, Jenny GJ, Bauer GF. The Context, Process, and Outcome Evaluation Model for Organisational Health Interventions. Biomed Res Int. 2015;2015:414832.

[37] Sen AK. Development as freedom. New York: Knopf; 1999.

[38] van der Klink JJ, Bultmann U, Burdorf A, Schaufeli WB, Zijlstra FR, Abma FI, Brouwer S, van der Wilt GJ. Sustainable employability-definition, conceptualization, and implications: A perspective based on the capability approach. Scand J Work Environ Health. 2016;42(1):71-9.

[39] Nielsen K. Organizational occupational health interventions: what works for whom in which circumstances? Occup Med (Lond). 2017;67(6):410-2.

[40] Nicholson PJ. Common mental disorders and work. Br Med Bull. 2018;126(1):113-21

[41] Jagosh J, Pluye P, Wong G, Cargo M, Salsberg J, Bush PL, Herbert CP, Green LW, Greenhalgh T, Macaulay AC. Critical reflections on realist review: insights from customizing the methodology to the needs of participatory research assessment. Res Synth Methods. 2014;5(2):131-41.

[42] Pawson R, Greenhalgh T, Harvey G, Walshe K. Realist review: a new method of systematic review designed for complex policy interventions. Journal of Health Services Research \& Policy. 2005;10(Suppl 1):21-34.

[43] Kirst M, O'Campo P. Realist review methods for complex health problems. Rethinking Social Epidemiology: Springer; 2012. pp. 231-45.

[44] Jagosh J, Pluye P, Macaulay AC, Salsberg J, Henderson J, Sirett E, Bush PL, Seller R, Wong G, Greenhalgh T, Cargo M, Herbert CP, Seifer SD, Green LW. Assessing the outcomes of participatory research: protocol for identifying, selecting, appraising and synthesizing the literature for realist review. Implement Sci. 2011;6:24.

[45] Emmel N, Greenhalgh J, Manzano A, Monaghan M, Dalkin S, editors. Doing realist research. London: Sage; 2018.

[46] Dalkin SM, Greenhalgh J, Jones D, Cunningham B, Lhussier M. What's in a mechanism? Development of a key concept in realist evaluation. Implement Sci. 2015;10:49. 
[47] Wong G, Greenhalgh T, Westhorp G, Buckingham J, Pawson R. RAMESES publication standards: realist syntheses. BMC Med. 2013;11:21.

[48] Moher D, Liberati A, Tetzlaff J, Altman DG. The PRISMA Group. Preferred reporting items for systematic reviews and meta-analyses: the PRISMA statement. PLoS Med. 2009;6(7):e10000097.

[49] Ouzzani M, Hammady H, Fedorowicz Z, Elmagarmid A. Rayyan - a web and mobile app for systematic reviews. Systematic Reviews. 2016;5(210).

[50] Pluye P, Gagnon MP, Griffiths F, Johnson-Lafleur J. A scoring system for appraising mixed methods research, and concomitantly appraising qualitative, quantitative and mixed methods primary studies in Mixed Studies Reviews. Int J Nurs Stud. 2009;46(4):529-46.

[51] Hong QN, Pluye P, Fábregues S, Bartlett G, Boardman F, Cargo M, Dagenais P, Gagnon M-P, Griffiths F, Nicolau B, O'Cathain A, Rousseau M-C, Vedel I. Mixed Methods Appraisal Tool (MMAT), version 2018, Registration of Copyright (\#1148552). Canadian Intellectual Property Office, Industry Canada. 2018.

[52] Jagosh J, Bush PL, Salsberg J, Macaulay AC, Greenhalgh T, Wong G, Cargo M, Green LW, Herbert CP, Pluye P. A realist evaluation of community-based participatory research: partnership synergy, trust building and related ripple effects. BMC Public Health. 2015;15:725.

[53] Lappalainen P, Kaipainen K, Lappalainen R, Hoffren H, Myllymaki T, Kinnunen ML, Mattila E, Happonen AP, Rusko H, Korhonen I. Feasibility of a personal health technology-based psychological intervention for men with stress and mood problems: randomized controlled pilot trial. JMIR Res Protoc. 2013;2(1):e1. 